\title{
Graft Polymerization of Vinyl Monomers from Inorganic Ultrafine Particles Initiated by Azo Groups Introduced onto the Surface
}

\author{
Norio Tsubokawa, Akira Kogure, Kazue Maruyama, \\ Yasuo Sone, and Masato SHIMOMURA* \\ Department of Material and Chemical Engineering, Niigata University, \\ 8050, Ikarashi 2-nocho, Niigata 950-21, Japan \\ * Advanced Technology Research Center, Technical Headquarters, \\ Mitsubishi Heavy Industries Ltd., 8-1, Sachiura l-chome, \\ Kanazawa-ku, Yokohama 236, Japan
}

(Received April 10, 1990)

\begin{abstract}
The radical graft polymerization of vinyl monomers from inorganic ultrafine particles, such as silica, titanium oxide, and ferrite, by use of azo groups introduced onto their surface was investigated. The introduction of azo groups onto the ultrafine particles was achieved by the reaction of glycidyl groups, which were introduced by the reaction of hydroxyl groups on the surface with 3-glycidoxypropyltrimethoxysilane, with 4,4'-azobis(4-cyanopentanoic acid). The amounts of azo groups introduced onto silica, titanium oxide, and ferrite were determined to be $0.07,0.05$, and $0.03 \mathrm{mmol} \mathrm{g}^{-1}$, respectively. The polymerization of vinyl monomers, such as methyl methacrylate (MMA), styrene, and $N$-vinylcarbazole (NVC), was found to be initiated by radicals formed by the decomposition of the azo groups. During the polymerization, the polymer was effectively grafted onto these surfaces through propagation from the surfaces; the percentage of grafting of polyMMA onto silica, titanium oxide, and ferrite reached to $45.1,42.8$, and $40.5 \%$, respectively. Polymer-grafted ultrafine particles produced stable dispersions in organic solvents. Furthermore, the dispersion of polymer-grafted ferrite in organic solvent was found to behave like a magnetic fluid.
\end{abstract}

KEY WORDS Inorganic Ultrafine Particle / Surface Grafting / Silica / Titanium Oxide / Ferrite / Vinyl Monomer / 4,4'-Azobis(4-cyanopentanoic acid) / 3-Glycidoxypropyltrimethoxysilane / Azo Group / Dispersibility /

The dispersibility of inorganic ultrafine particles in organic solvents or polymer matrix is remarkably improved by grafting of polymers onto these surfaces, because the grafted polymer chains on the surface interfere with the aggregation of ultrafine particles and increase the affinity of the surface for solvents. ${ }^{1,2}$

In the preceding paper, we reported the radical graft polymerization of acrylamide (AAm) from ultrafine silica initiated by a redox system consisting of ceric ion and reducing groups, such as alcoholic hydroxyl, amono and mercapto groups, introduced onto the surface. ${ }^{3}$
In the polymerization, polyAAm-grafted silica with a higher percentage of grafting was obtained, because the polymer propagates through radicals produced by the redox reaction of the reducing groups with ceric ion.

Furthermore, we demonstrated that the graft polymerization of vinyl monomers is initiated by peroxyester ${ }^{4}$ and azo groups ${ }^{5}$ introduced onto the carbon black surface. During the polymerization, vinyl polymers propagate through radicals produced on the surface by the decomposition of these groups to give polymer-grafted carbon black with a higher percentage of grafting. 
In the present paper, the introduction of azo groups onto inorganic ultrafine particles, such as silica, ferrite, and titanium oxide, and the graft polymerization of vinyl monomers ini- tiated by azo groups on the surfaces were investigated (eq 1). Furthermore, the stability of dispersion of vinyl polymer-grafted ultrafine particles was examined.

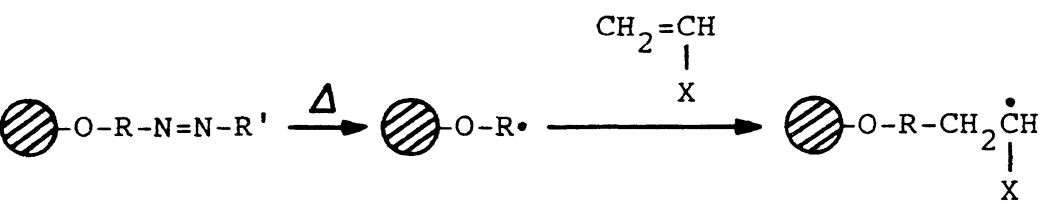

$$
\begin{aligned}
& \longrightarrow-\mathrm{O}+\mathrm{CH}_{2}-{ }_{x}^{\mathrm{CH}+}{ }_{\mathrm{n}}-\left.\mathrm{CH}_{2}\right|_{\mathrm{X}} ^{\dot{\mathrm{C}} \mathrm{H}}
\end{aligned}
$$

\section{EXPERIMENTAL}

\section{Materials}

Ultrafine silica, titanium oxide, and ferrite used were Aerosil 200 (Nippon Aerosil Co., Japan), STT-30 (Titan Kogyo K.K., Japan) and ultrafine nickel zinc ferrite (Sumitomo Cement Co., Ltd., Japan), respectively. The BET specific surface area, particle size, and the content of hydroxyl group on the surface of these ultrafine particles are shown in Table I. The content of hydroxyl groups was determined by measuring volumetrically the amount of ethane evolved by the reaction with triethylaluminum. ${ }^{6}$ The ultrafine particles were dried in vacuo at $110^{\circ} \mathrm{C}$ before use.

Methyl methacrylate (MMA) was washed with dilute alkali solution, dried over sodium

Table I. Properties of ultrafine silica, titanium oxide, and ferrite

\begin{tabular}{lcccc}
\hline & $\begin{array}{c}\text { BET surface } \\
\text { Ultrafine } \\
\text { particle }\end{array}$ & $\begin{array}{c}\text { Particle } \\
\text { size }\end{array}$ & & OH group \\
\cline { 2 - 3 } & $\mathrm{m}^{2} \mathrm{~g}^{-1}$ & $\mathrm{~nm}$ & $\mathrm{mmol} \mathrm{g}^{-1}$ \\
Silica $^{\mathrm{a}}$ & 200 & 16 & 1.37 \\
Titanium oxide $^{\mathrm{b}}$ & $90-150$ & 120 & 0.77 \\
Ferrite $^{\mathrm{c}}$ & 110 & 15 & 0.50 \\
\hline
\end{tabular}

a Aerosil 200 (Nippon Aerosil Co.).

b STT-30 (Titan Kogyo K.K.).

c Ultrafine nickel zinc ferrite (Sumitomo Cement Co., Ltd.). sulfate, and distilled under reduced pressure. Styrene was also washed with dilute alkali solution, dried over barium oxide, and distilled under reduced pressure. Acrylonitrile (AN) was distilled twice before use. $N$-Vinylcarbazole (NVC) was recrystallized from hexane and dried in vacuo at room temperature.

3-Glycidoxypropyltrimethoxysilane (GPS) was obtained from Kanto Chemical Co., Inc., Japan and used without further purification. 4,4'-Azobis(4-cyanopentanoic acid) (ACP) obtained from Aldrich Chemical Co., Inc. was recrystallized from methanol.

Dimethyl sulfoxide (DMSO) was dried over calcium hydride and distilled twice under reduced pressure. Other solvents and reagents were used after ordinary purification.

\section{Introduction of Azo Groups onto Ultrafine Particles}

The introduction of azo groups onto ultrafine particles was achieved by the reaction of glycidoxy groups introduced onto the surface with ACP.

Glycidoxy groups were introduced onto the surface by the reaction of hydroxy groups on the surface with GPS. A typical example is as follows. Into a flask, $10.0 \mathrm{~g}$ of ultrafine particles and $100 \mathrm{~cm}^{3}$ of $5 \%$ toluene solution of GPS were charged. The reaction mixture was refluxed for $8 \mathrm{~h}$. After the reaction, ultrafine particles were extracted with methanol using a 
Soxhlet apparatus.

The reaction of glycidoxy groups on the surface with ACP was carried out as follows. Into a flask, $3.0 \mathrm{~g}$ of GPS-treated particles, $50 \mathrm{~cm}^{3}$ of DMSO, $0.5 \mathrm{~g}$ of ACP, and $\alpha$-picoline were charged. The reaction mixture was stirred with a magnetic stirrer for $5 \mathrm{~h}$ at $50^{\circ} \mathrm{C}$. After the reaction, the resulting particles were washed with methanol and dried in vacuo at room temperature. The azo group-introduced ultrafine particles were stored below $-10^{\circ} \mathrm{C}$ in the dark.

\section{Polymerization Procedures}

Into a polymerization tube, $0.30 \mathrm{~g}$ of ultrafine particles and $10.0 \mathrm{~cm}^{3}$ of monomer were charged. The tube was cooled in a liquid nitrogen bath, thawed three times with nitrogen, and sealed. the polymerization was conducted at $70^{\circ} \mathrm{C}$ with shaking. When NVC was used as monomer, the polymerization was carried out using $0.30 \mathrm{~g}$ of ultrafine particles, $3.0 \mathrm{~g}$ of NVC, and $10 \mathrm{~cm}^{3}$ of dioxane. After a definite time, the reaction mixture was poured into a large excess of methanol and the precipitate was dried in vacuo at $40^{\circ} \mathrm{C}$. The conversion was determined by the following equation:

$$
\begin{aligned}
& \text { Conversion }(\%)= \\
& \frac{\text { Product }(\mathrm{g})-\text { Ultrafine particle }(\mathrm{g})}{\text { Monomer used }(\mathrm{g})} \times 100
\end{aligned}
$$

\section{Percentage of Grafting}

The product was dispersed in a solvent for the polymer and the dispersion was centrifuged at $1.0 \times 10^{4} \mathrm{rpm}$ until the ultrafine particles precipitated completely. The particles were extracted using a Soxhlet apparatus until no polymer was eluted in the refluxing solvent. The percentage of grafting was calculated as follows:

$$
\begin{aligned}
& \text { Percentage of grafting }(\%)= \\
& \frac{\text { Polymer grafted }(\mathrm{g})}{\text { Ultrafine particles used }(\mathrm{g})} \times 100
\end{aligned}
$$

\section{Stability of Dispersion of Polymer-Grafted Particle}

Polymer-grafted ultrafine particles $(0.5 \mathrm{~g})$ were dispersed in $100 \mathrm{~cm}^{3}$ of tetrahydrofuran (THF). After a definite time, $5.0 \mathrm{~cm}^{3}$ of the dispersion liquid were taken out with a pipet and the ultrafine particles dispersed were determined. The stability of dispersion was estimated from the content of particles in the dispersion:

Content of particles in the dispersion (\%)=

$$
\frac{A}{B} \times 100
$$

$A$, ultrafine particles in dispersed phase (g); $B$, ultrafine particles used $(\mathrm{g})$.

\section{Infrared Spectra}

The infrared spectra of polymer-grafted ultrafine particles were recorded on a Hitachi Infrared Spectrophotometer Model 270-30 using $\mathrm{KBr}$ pellets.

\section{RESULTS AND DISCUSSION}

\section{Introduction of Azo Group onto Ultrafine Particles}

The introduction of azo groups onto the surface of ultrafine particles was achieved by reaction of glycidoxy groups introduced by the reaction of surface hydroxyl groups with GPS (eq 2), with ACP in DMSO at $50^{\circ} \mathrm{C}$ for $5 \mathrm{~h}$ (eq 3 ). It is not necessary to take into account the decomposition of ACP during the reaction because the half-lives of ACP is reported to be about $10000 \mathrm{~min} .{ }^{7}$ In the reaction, $\alpha$-picoline was used as catalyst.

Table II. The amounts of azo groups introduced onto silica, titanium oxide, and ferrite surface

\begin{tabular}{lc}
\hline Ultrafine particle & Azo group $/ \mathrm{mmol} \mathrm{g}^{-1}$ \\
\hline Silica & 0.07 \\
Titanium oxide & 0.05 \\
Ferrite & 0.03 \\
\hline
\end{tabular}


The amounts of azo groups introduced onto were determined by elemental the surface of silica, titanium oxide, and ferrite

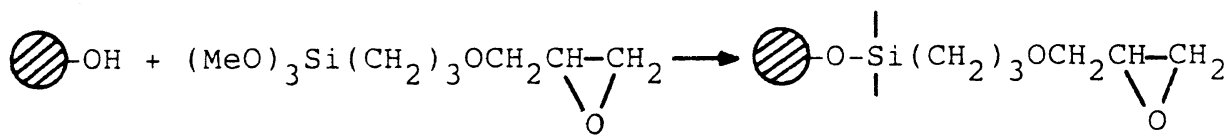

$$
\begin{aligned}
& 1 \\
& 1+\operatorname{HOOC}\left(\mathrm{CH}_{2}\right) \overbrace{\mathrm{CN}}^{\mathrm{Me}} \overbrace{\mathrm{CN}}^{\mathrm{Me}} \overbrace{\mathrm{C}}^{\mathrm{Me}}\left(\mathrm{CH}_{2}\right)_{2} \mathrm{COOH} \\
& \text { Q }
\end{aligned}
$$

analysis of nitrogen. The results are summarized in Table II.

Table II shows that azo groups can be definitely introduced onto silica, titanium oxide, and ferrite surfaces by the reaction of glycidoxy groups with ACP. The amounts of azo groups introduced onto the surface increased with hydroxyl group content of ultrafine particles.

Initiating Activity of Azo Groups Introduced onto Silica Surface

The polymerization of MMA was carried out in the presence of silica having azo group (Silica-Azo) at $70^{\circ} \mathrm{C}$ and the initiating activity of the azo groups on the surface was examined. The results are shown in Table III.

As shown in Table III, the thermal

Table III. Polymerization of MMA in the presence of various treated silicas ${ }^{\mathbf{a}}$

\begin{tabular}{lcc}
\hline Silica & Conversion/\% & Grafting/\% \\
\hline None & 1.6 & - \\
Untreated & 1.4 & 0 \\
ACP-adsorbed & 1.7 & 0 \\
Silica-Azo & 34.0 & 45.1 \\
\hline
\end{tabular}

a Silica, $0.30 \mathrm{~g}$; MMA, $10.0 \mathrm{~cm}^{3} ; 70^{\circ} \mathrm{C} ; 2 \mathrm{~h}$. polymerization of MMA proceeded even in the absence of initiator, though the rate of polymerization was considerably low. Untreated silica has no ability to initiate the polymerization. But in the presence of SilicaAzo, the polymerization of MMA was found to be initiated.

To ensure initiation by azo groups on the silica surface, the initiating activity of ACPadsorbed silica was evaluated. As shown in Table III, ACP-adsorbed silica failed to initiate the polymerization. This suggest that ACP adsorbed on the silica surface during the introduction of azo groups is removed completely by washing with methanol.

Based on the above results, it is concluded that Silica-Azo has ability to initiate the polymerization of MMA.

\section{Proof of Grafting onto Silica}

Figure 1 shows the time vs. conversion curve and time $v s$. percentage of grafting curve for the polymerization of MMA initiated by Silica-Azo. It is apparent that polyMMA is grafted onto the silica surface and percentage of grafting reached about $45 \%$ after $1.5 \mathrm{~h}$.

To evaluate the adsorption of polyMMA onto the silica, untreated silica was mixed with 


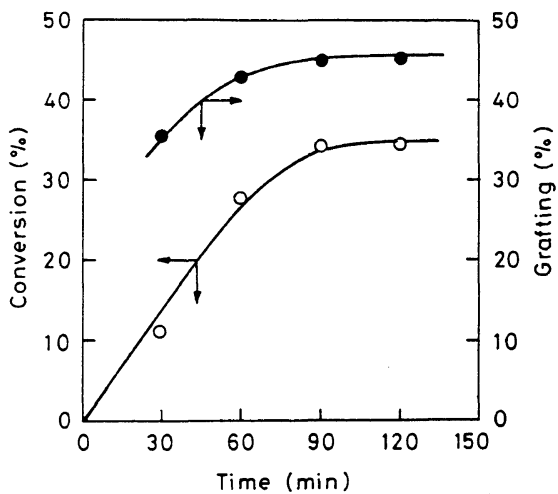

Figure 1. Graft polymerization of MMA initiated by azo groups introduced onto silica surface. Silica-Azo, $0.30 \mathrm{~g}$; MMA, $10.0 \mathrm{~cm}^{3} ; 70^{\circ} \mathrm{C}$.

polyMMA in $\mathrm{THF}$ at $30^{\circ} \mathrm{C}$ for $2 \mathrm{~h}$. The amount of the polymer remaining on the surface after extracting with THF was determined to be $2.0 \%$. This value is much less than that of the polymer grafted onto the surface. This indicates that it is not necessary to take into account the effect of adsorption of polyMMA during the polymerization on the grafting.

The azo group onto the silica surface is considered to produce both surface radicals and 4-cyanopentanoic acid radicals by its decomposition. The former initiates the graft polymerization from the surface, but the latter produces ungrafted polymer. The grafting efficiency, that is, the ratio of grafted polymer to the total polymer formed, is very low, about $4 \%$ in the above graft polymerization. This indicates that the polymerization of MMA initiated by 4-cyanopentanoic acid radicals preferentially proceeds to give ungrafted polymer. The grafted polymer chains may thus interfere with the propagation of polymer from radicals formed on the surface.

Figure 2 shows infrared spectra of untreated and polyMMA-grafted silica. In the infrared spectrum of polyMMA-grafted silica, a new absorption at $1735 \mathrm{~cm}^{-1}$, assigned to ester bond of polyMMA was observed. On the other hand, the infrared spectrum of polyMMAadsorbed silica was the same as that of an

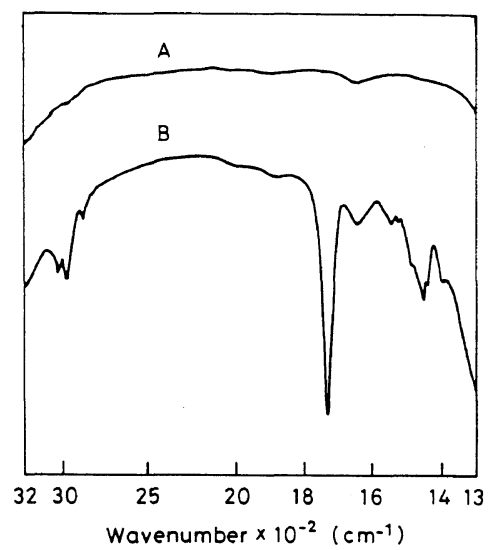

Figure 2. IR spectra of (A) untreated and (B) polyMMAgrafted silica (percentage of grafting $=20.6 \%$ ).

Table IV. Polymerization of MMA initiated by azo groups introduced onto silica, titanium oxide, and ferrite ${ }^{\mathrm{a}}$

\begin{tabular}{lccccc}
\hline \multirow{2}{*}{ Ultrafine particle } & Time & & Conversion & & Grafting \\
\cline { 2 - 2 } \cline { 5 - 6 } \cline { 5 - 5 } & $\mathrm{h}$ & & $\%$ & & $\%$ \\
\hline Silica-Azo & 2 & & 34.0 & & 45.1 \\
Titanium oxide-Azo & 9 & & 8.7 & & 40.0 \\
Ferrite-Azo & 9 & & 7.5 & & 38.7 \\
\hline
\end{tabular}

a Ultrafine particle, $0.30 \mathrm{~g} ; \mathrm{MMA}, 10.0 \mathrm{~cm}^{3} ; 70^{\circ} \mathrm{C}$.

untreated one.

Therefore, it is concluded that polyMMA is grafted onto silica through the propagation of the polymer from the radical formed by the decomposition of azo groups on the surface as shown in eq 1.

\section{Grafting from Various Ultrafine Particles}

The graft polymerization of MMA initiated by azo groups introduced onto titanium oxide (Titanium oxide-Azo) and ferrite (Ferrite-Azo) at $70^{\circ} \mathrm{C}$ was compared with that of Silica-Azo. The results are summarized in Table IV.

It is apparent that Titanium oxide-Azo and Ferrite-Azo also have ability to initiate the polymerization of MMA and polyMMA is effectively grafted onto these surfaces. The rate of polymerization and percentage of grafting 
Table V. Polymerization of several vinyl monomers initiated by azo groups introduced onto silica and ferrite surface

\begin{tabular}{|c|c|c|c|c|}
\hline \multirow{2}{*}{$\begin{array}{l}\text { Ultrafine } \\
\text { particle }\end{array}$} & \multirow{2}{*}{ Monomer } & \multirow{2}{*}{$\frac{\text { Time }}{\mathrm{h}}$} & \multirow{2}{*}{$\frac{\text { Conversion }}{\%}$} & \multirow{2}{*}{$\frac{\text { Grafting }}{\%}$} \\
\hline & & & & \\
\hline Silica-Azo & $\mathrm{MMA}^{\mathrm{a}}$ & 2 & 34.0 & 45.1 \\
\hline Silica-Azo & $\mathrm{AN}^{\mathrm{a}}$ & 6 & 28.4 & 35.8 \\
\hline Silica-Azo & $\mathrm{NVC}^{\mathbf{b}}$ & 6 & 57.7 & 33.8 \\
\hline Silica-Azo & Styrene $^{\mathbf{a}}$ & 6 & 5.6 & 26.2 \\
\hline Ferrite-Azo & $\mathrm{MMA}^{\mathrm{a}}$ & 9 & 7.5 & 38.7 \\
\hline Ferrite-Azo & $\mathrm{NVC}^{\mathrm{b}}$ & 9 & 23.8 & 30.8 \\
\hline Ferrite-Azo & Styrene ${ }^{a}$ & 9 & 4.3 & 20.0 \\
\hline
\end{tabular}

a Ultrafine particle, $0.30 \mathrm{~g}$; monomer, $10.0 \mathrm{~cm}^{3} ; 70^{\circ} \mathrm{C}$.

b Ultrafine particle, $0.30 \mathrm{~g}$; NVC, $3.0 \mathrm{~g}$; dioxane, $10.0 \mathrm{~cm}^{3} ; 70^{\circ} \mathrm{C}$.

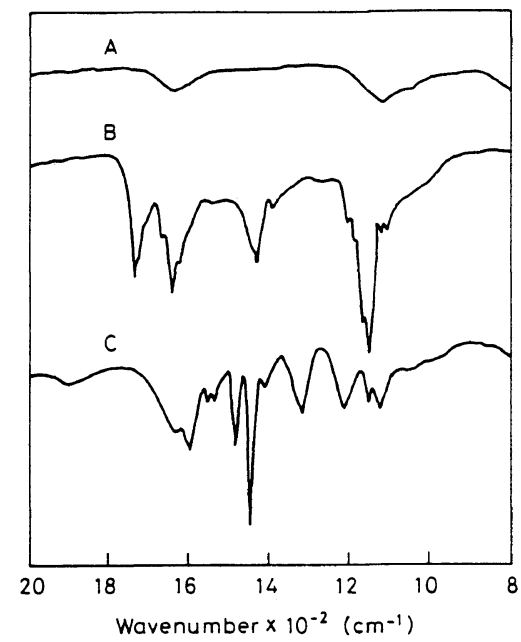

Figure 3. IR spectra of (A) untreated, (B) polyMMAgrafted (percentage of grafting $=38.7 \%$ ), and $(C)$ polyNVC-grafted ferrite (percentage of grafting $=30.8 \%$ ).

of polyMMA were found to increase in the following order: ferrite $<$ titanium oxide $<$ silica. This order is in agreement with that of increasing azo group content.

\section{Grafting of Several Vinyl Polymers onto Ultrafine Particles}

The graft polymerization of several monomers, such as AN, NVC, and styrene, initiated by azo groups introduced onto silica and ferrite

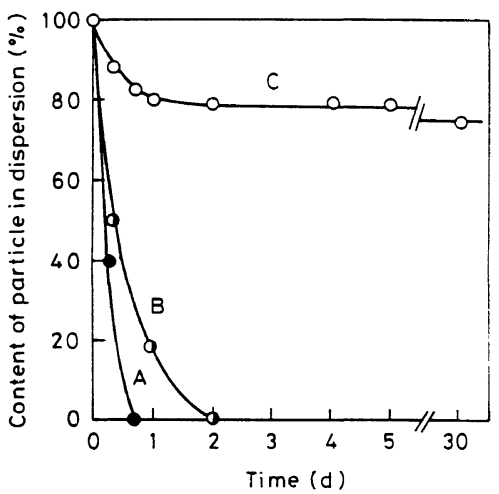

Figure 4. Stability of dispersion of (A) ungrafted, (B) polyMMA-adsorbed, and (C) polyMMA-grafted silica (percentage of grafting $=45.1 \%$ ) in THF.

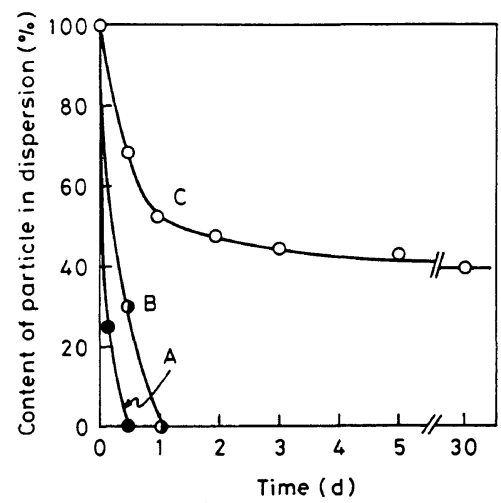

Figure 5. Stability of dispersion of (A) ungrafted, (B) polyMMA-adsorbed, and (C) polyMMA-grafted ferrite (percentage of grafting $=38.7 \%$ ) in THF.

surfaces was compared with that of MMA. The results are summarized in Table V. Figure 3 shows infrared spectra of ungrafted, polyMMA-grafted, and polyNVC-grafted ferrite.

Table $\mathrm{V}$ and Figure 3 indicate that the polymerization of AN, NVC, and styrene is initiated by azo groups introduced onto the surfaces of silica and ferrite and the corresponding polymers are grafted onto these surfaces. Therefore, ultrafine particles containing azo groups are useful for the grafting of various vinyl monomers onto the surfaces.

\section{Stability of Dispersion of Polymer-Grafted Ultrafine Particles \\ The stability of dispersion of polyMMA-}


grafted silica and ferrite in THF was compared with that of untreated ones. The results on polyMMA-grafted silica and polyMMA-graftted ferrite are shown in Figures 4 and 5, respectively.

Ungrafted silica and ferrite precipitated completely after $24 \mathrm{~h}$. The stability of dispersion of silica and ferrite was scarcely improved by the adsorption of polyMMA. On the contrary, polyMMA-grafted silica and ferrite afforded stable dispersions in THF. The reason for that the grafted polymer chains on the surface interfere with the aggregation of ultrafine particles. In addition, polystyrenegrafted or polyNVC-grafted ultrafine particles also dispersed stably in various organic solvents.

Accordingly, the dispersibility of ultrafine particles in organic solvents was remarkably improved by grafting of polymer onto the surface.

When a magnet was brought to the dispersion of ungrafted ferrite in THF, only ferrite particles were attracted by the magnet without the dispersion liquid. But in the case of polyMMA-grafted ferrite dispersion, polyMMA-grafted ferrite was attracted by a magnet with the dispersion liquid. Therefore, the dispersion of polymer-grafted ferrite in the polymer solvents behaves like a magnetic fluid.

Acknowledgments. This work was partly supported by Ministry of Education, Science, and Culture of Japan under Grant to N.T., which is gratefully acknowledged.

\section{REFERENCES}

1. R. Kroker, M. Schneider, and K. Hamann, Progr. Org. Coatings, 1, 23 (1972).

2. R. Laible and K. Hamann, Adv. Colloid Interface Sci., 13, 65 (1980).

3. N. Tsubokawa, K. Maruyama, Y. Sone, and M. Shimomura, Polym. J., 21, 475 (1989).

4. N. Tsubokawa, K. Fujiki, and Y. Sone, Polym. J., 20, 213 (1988)

5. N. Tsubokawa, K. Fujiki, and Y. Sone, Polym. J., 22, 661 (1990).

6. S. Matsuda and S. Okazaki, Nippon Kagaku Kaishi, 1287 (1986).

7. W. J. Bengough and H. W. Melvile, Proc. Roy. Soc. (London), Ser. A, 249. 445 (1959). 